\title{
ISAR Imaging of maneuvering Targets based on a new Algorithm Modified Fast Chirp Fourier Transform for Cubic Chirps
}

\author{
Anitha $\mathrm{G}^{1}$, Ranga Rao K.S $\mathrm{S}^{2}$ \\ ${ }^{1}$ Department of ECE, GIT, GITAM University \\ anithaguttavelli@gmail.com, \\ ${ }^{2}$ Dept.of ECE, Anits, Visakhapatnam \\ srrkolluri@gmail.com
}

\begin{abstract}
Inverse Synthetic Aperture Radar (ISAR) imaging of targets with complex motions can be modeled as cubic chirps. Modified Discrete Chirp Fourier Transform (MDCFT) is used to process the cubic signal to obtain ISAR image in two dimensions (2D). In this paper, a new algorithm named Modified Fast Chirp Fourier Transform (MFCFT) is proposed. This algorithm gives accurate results as, and the computation time is very much reduced, compared to MDCFT. The simulation is carried out for ship and Airplane targets with different scattering points. The obtained results show an enhancement both in terms of resolution and computation time in the proposed method hence named Modified Fast chirp Fourier Transform.
\end{abstract}

Keywords: ISAR, MDCFT, MFCFT, RD, RID,RIC.

\section{I.INTRODUCTION}

ISAR imaging is used to produce the high resolution images of moving targets. The focused image of targets with uniform motion is obtained by conventional Range Doppler (RD) method [1]. When targets have high complex motions then they can be characterized by second order phase terms and Range Instantaneous Doppler (RID) is used to obtain the image [2]. Several available ISAR imaging approaches have been previously discussed like FFT, Time frequency transform [3,4], Fractional Fourier transform[5], S-method, Clean technique, Wigner Ville distribution[6]. For targets with very high complex motions such as significant angular motions, like, pitch, roll and yaw, the radar azimuth echoes must be modeled as cubic chirp signals and to obtain the image, Range Instantaneous Chirp (RIC) is used. Therefore, the signal processing of cubic chirps is very important in the ISAR imaging. Presently several algorithms are available for cubic chirps like TC Dechirp clean technique, Product High order Matched Phase Transform (PHMT) [7], Discrete chirp Fourier transform, Modified discrete chirp Fourier transform [8-10]. High quality ISAR images can be effectively obtained via the proposed imaging method both by means of resolution and computation time.

\section{II.MODELING FOR CUBIC CHIRP}

When very high maneuvering of target takes place, then the phase of the return signal is represented in terms of higher order phase terms or cubic phase terms. The transmitted LFM signal is represented by [11]

$$
\begin{aligned}
& S\left(t_{1}, t_{2}\right)=\operatorname{rect}\left(\frac{t_{1}}{T}\right) \exp \left(j 2 \pi\left(f(t)+\frac{1}{2} \gamma(t)^{2}\right)\right) \\
& \text { where } \operatorname{rect}\left(\frac{t_{1}}{T}\right)=\left\{\begin{array}{l}
1,\left|\left(\frac{t_{1}}{T}\right)\right| \leq \frac{1}{2} \\
0,\left|\left(\frac{t_{1}}{T}\right)\right|>\frac{1}{2}
\end{array}\right\}
\end{aligned}
$$

Where.f, $\gamma$ and $\mathrm{T}$, denote the carrier frequency, chirp rate and pulse width respectively, $\mathrm{t}$ is the full time given by $t_{1}+t_{2}, t_{1}$ being the fast time and $t_{2}$, the slow time. $t_{2}$ is given by $n / p r f$ and $n=0,1, \ldots N$. Here, prf is pulse repetition frequency and $\mathrm{N}$ is the number of accumulating pulses. The received echo signal is given by

$$
S_{i}\left(t_{1}, t_{2}\right)=\sigma_{i} \operatorname{rect}\left(\frac{t_{1}-2 R_{i}\left(t_{2}\right) / c}{T}\right) \exp \left(j 2 \pi\left(f\left(t-2 R_{i}\left(t_{2}\right) / c\right)+\frac{1}{2} \gamma\left(t-2 R_{i}\left(t_{2}\right) / c\right)^{2}\right)\right)
$$

where $R_{i}\left(t_{2}\right)$ is the distance between $i^{\text {th }}$ scatter and Radar at time $t_{2}$ and $\sigma_{i}$ represents the reflection coefficient. For this received signal keystone formatting is applied to remove the migration through range cell [12]. Assume that if the number of scatters in a certain range bin is $\mathrm{I}$, the azimuth echoes corresponding to this range bin is expressed as the cubic phase signal [13-18]. The azimuth echo signal is written as 


$$
\begin{aligned}
& S^{\prime}\left(t_{2}\right)=\sum_{i=1}^{I} A_{i} \exp \left[j\left(\theta_{i}+2 \pi\left(f_{i} t_{2}+\frac{1}{2} \gamma_{i} t_{2}^{2}+\frac{1}{6} \beta_{i} t_{2}^{3}\right)\right)\right] \\
& \theta_{i}=-\left(\frac{4 \pi}{\lambda}\right) R_{i}\left(t_{0}\right) \\
& f_{i}=-\frac{2}{\lambda}\left(\omega \cdot r^{T}\right) \\
& \gamma_{i}=-\frac{2}{\lambda}\left(\alpha \cdot r^{T}\right) \\
& \beta_{i}=-\frac{2}{\lambda}\left(\zeta \cdot r^{T}\right)
\end{aligned}
$$

where $A_{i}$ denote the amplitude, $\theta_{i}$ the initial phase, $f_{i}$ the center frequency, $\alpha_{i}$ the chirp rate, and $\beta_{i}$ is derivative of chirp rate of azimuth echoes associated with the $i^{\text {th }}$ scatter respectively. $R_{i}\left(t_{0}\right)$ is initial range at time $t_{0}$. Therefore, the discrete expression of $S^{\prime}\left(t_{2}\right)$ is $S(n)$ and is given by

$$
S(n)=\sum_{i=1}^{I} A_{i} W_{N}^{f_{i} n+\left(\frac{\gamma_{i}}{N}\right) n^{2}+\left(\frac{\beta_{i}}{N^{2}}\right) n^{3}}
$$

where

$$
\begin{aligned}
W_{N} & =\exp \left(\frac{-j 2 \pi}{N}\right), A_{i}=a_{i} \exp \left(-j \frac{4 \pi}{\lambda}\right) R_{i}\left(t_{0}\right), f_{i}=\left(\frac{2 \omega r^{T}}{\lambda}\right)\left(\frac{N}{p r f}\right) \\
\gamma_{i} & =\left(\frac{\alpha r^{T}}{\lambda}\right)\left(\frac{N^{2}}{p r f}\right), \beta_{i}=\left(\frac{\zeta r^{T}}{3 \lambda}\right)\left(\frac{N^{3}}{p r f^{2}}\right),
\end{aligned}
$$

\section{III.ALGORITHMS FOR RIC}

To process the received echo signal for cubic chirps after MTRC, some transforms has to be used in order to obtain the final ISAR image in 2D. Discrete chirp Fourier transform and MDCFT have been used in the recent times to obtain the image. Following sections detail the three methods including the proposed one i.e., DCFT, MDCFT and MFCFT. A comparison of the three methods is also presented.

\section{A. DCFT}

DCFT was developed for quadratic terms and extended for cubic chirps which is represented by [11]

$$
\begin{gathered}
X(k, l, m)=\frac{1}{\sqrt{N}} \sum_{n=0}^{N-1} x(n) W_{N}^{k n+n^{2}+m n^{3}} \\
0 \leq k, l, m \leq N-1
\end{gathered}
$$

where $\mathrm{x}(\mathrm{n})$ is chirp signal having signal length $\mathrm{N}$ and $\mathrm{k}, 1$ and $\mathrm{m}$ represent the constant frequency, chirp rate and derivative of chirp rate respectively. If the signal parameters are not integers or not very close to integers then, DCFT will suffer from the picket-fence effect. In DCFT since $\mathrm{k}, 1, \mathrm{~m}$ values lies in between 0 to $\mathrm{N}-1$, the parameter estimation will not be accurate. Depending on the basic properties of DCFT, if $\mathrm{N}$ is a prime number then only the peak is clearly identified and if it is not prime then it leads to picket fence output.

For example consider the two cases.

Case (i). If $\mathrm{k}=36, \mathrm{l}=98, \mathrm{~m}=25$ and $\mathrm{N}=127$ (prime number) then the output for DCFT is shown in Fig.1. 


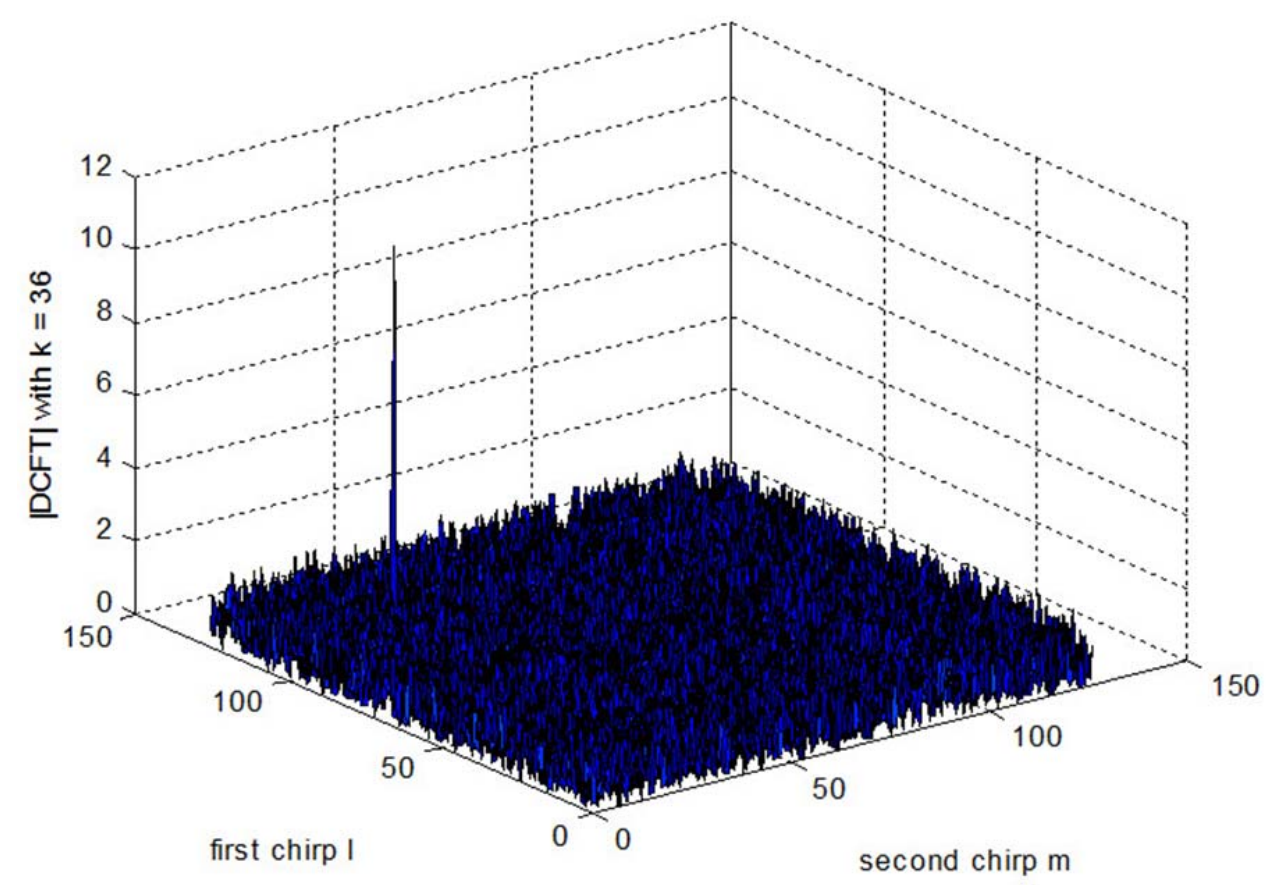

Fig.1. Three dimensional plot for DCFT when $\mathrm{N}=127$

Case (ii). If $\mathrm{k}=36, \mathrm{l}=98, \mathrm{~m}=25$ and $\mathrm{N}=128$ (not prime number) then the picket fence output is observed for DCFT in Fig.2.

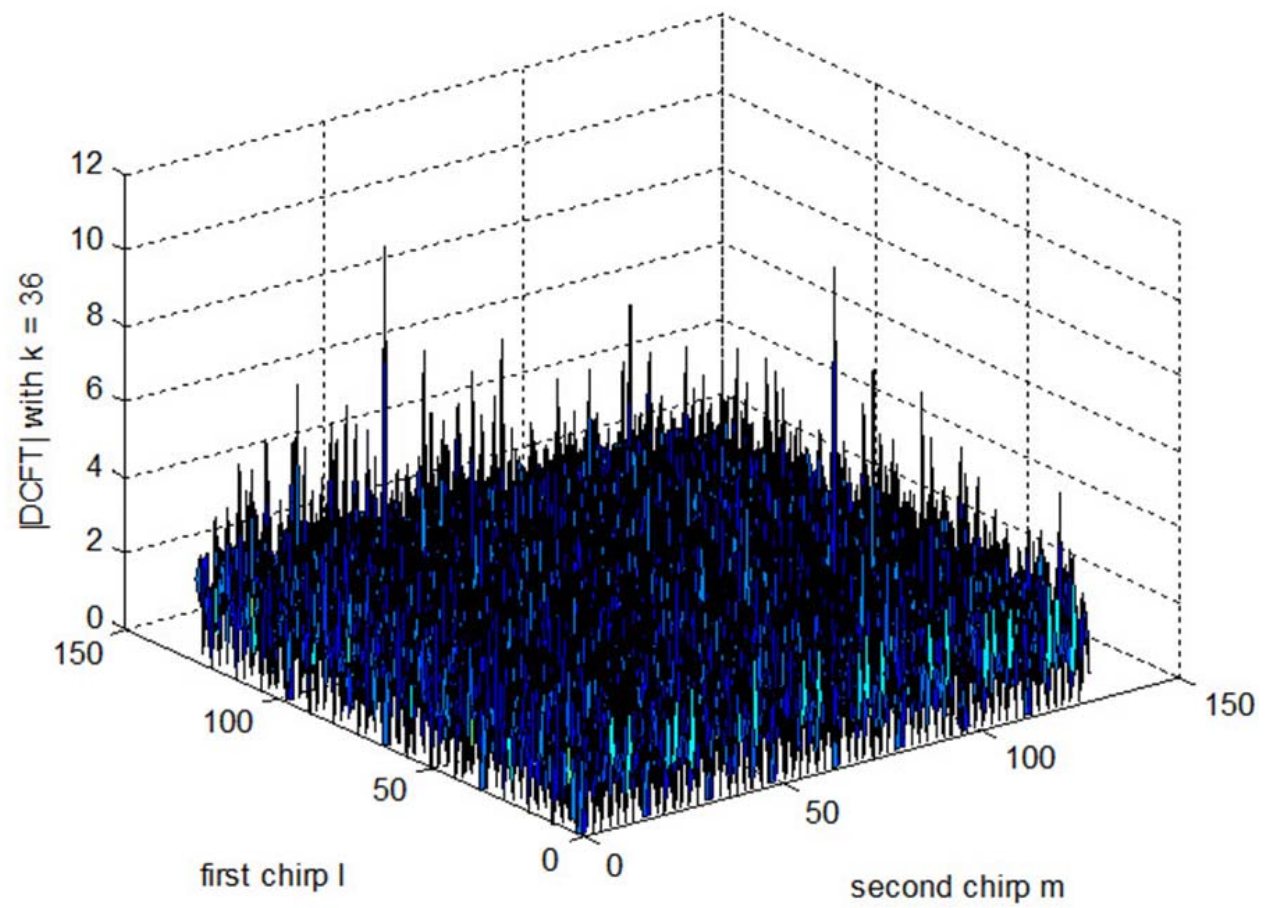

Fig.2 Three dimensional plot for DCFT for $\mathrm{N}=128$

From above Figs.1\&2, one can say that DCFT has a limitation in identifying the peak if $\mathrm{N}$ is non prime value. To avoid the severe picket-fence effect and increase the accuracy of parameter estimation, MDCFT is proposed.

B. MDCFT

MDCFT for cubic chirp signals is given by $[11,19,20]$

$$
\begin{gathered}
X(k, l, m)=\frac{1}{\sqrt{N}} \sum_{n=0}^{N-1} x(n) W_{N}^{k n+(l / N) n^{2}+\left(m / N^{2}\right) n^{3}} \\
0 \leq k, l, m \leq N-1
\end{gathered}
$$


The parameter estimation in MDCFT is from $\mathrm{k} \epsilon(0,1, \ldots \mathrm{N}-1), 1 \mathrm{\epsilon}(0,1 / \mathrm{N}, \ldots(\mathrm{N}-1) / \mathrm{N}), \mathrm{m} \epsilon\left(0,1 / \mathrm{N}^{2}, \ldots(\mathrm{N}-1) /\right.$ $\mathrm{N}^{2}$ ). Therefore, as the accuracy increases, a shorter near the peak sample interval can be obtained, and MDCFT will not suffer from picket-fence effect. This is verified with the below example.

If $\mathrm{k}=36, \mathrm{l}=98, \mathrm{~m}=25$ and $\mathrm{N}=128$ then the output for MDCFT is shown in Fig.3. From Fig.3, it can be said that the peak is clearly identified.

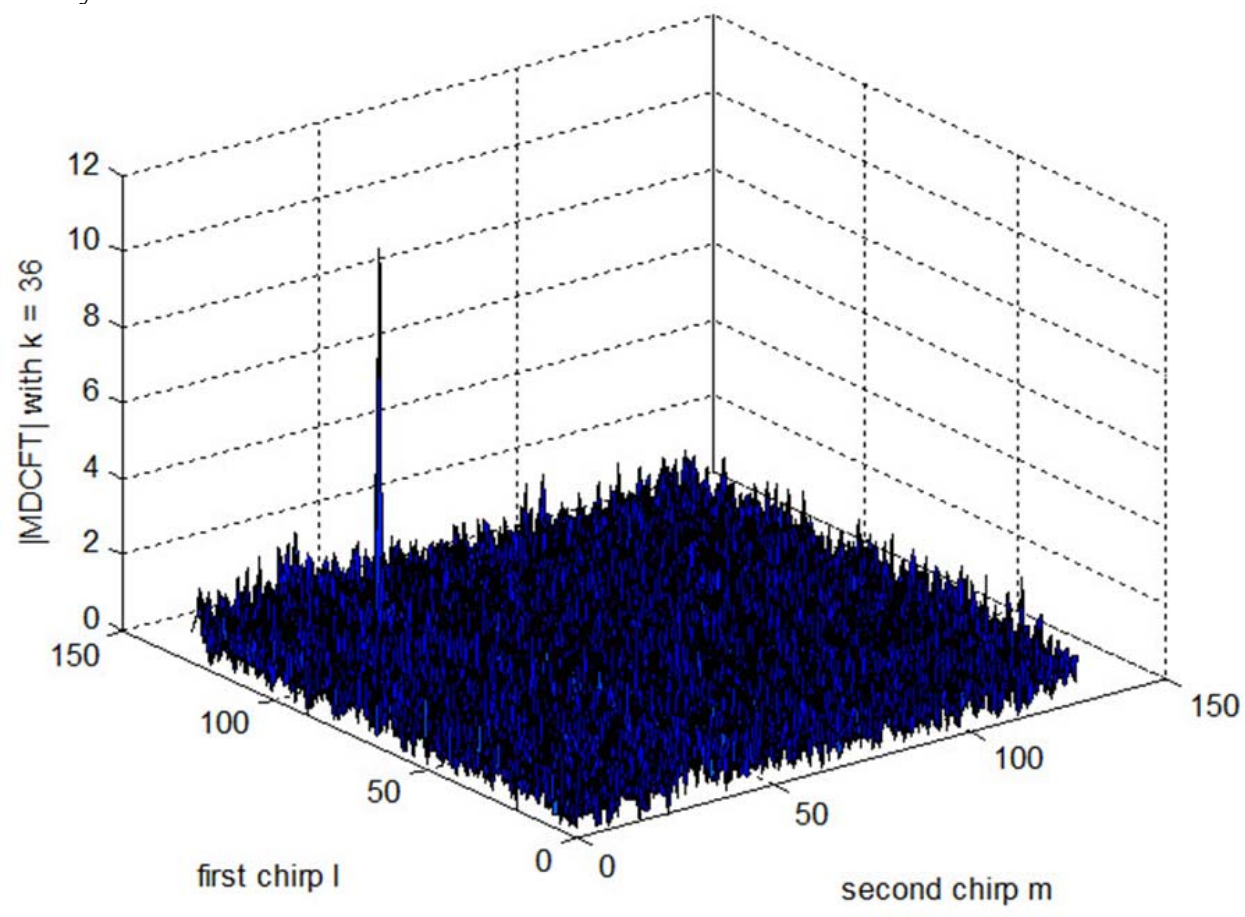

Fig.3.Three dimensional plot for MDCFT when $\mathrm{N}=128$

The ISAR imaging based on MDCFT for cubic chirps is implemented with the following steps as follows:

i. $\quad$ After applying MDCFT to (7) i.e., s(n) in each range bin, the equation is given by

$$
S(k, l, m)=\frac{1}{\sqrt{N}} \sum_{n=0}^{N-1} s(n) W_{N}^{k n+(l / N) n^{2}+\left(m / N^{2}\right) n^{3}}
$$

where $\mathrm{S}(\mathrm{k}, \mathrm{l}, \mathrm{m})$ is the MDCFT of $\mathrm{s}(\mathrm{n})$.

ii. The projection of $\mathrm{S}(\mathrm{k}, \mathrm{l}, \mathrm{m})$ will produce a Doppler image in $3 \mathrm{D}$ with center frequency $\mathrm{f}_{\mathrm{i}}$.

iii. Doppler image $\mathrm{I}_{\mathrm{D}}(\mathrm{k})$ can be obtained by the energy accumulation along axes of 1 and $\mathrm{m}$ given by

$$
I_{D}(k)=\left|\sum_{l, k} S(k, l, m)\right|
$$

In this Doppler image $\mathrm{I}_{\mathrm{D}}(\mathrm{k})$ all the main lobes of $\mathrm{S}(\mathrm{k}, 1, \mathrm{~m})$ are clearly identified.

iv. Now the Doppler images of all range bins are added to form the Range Doppler image of the target for cubic chirp signal.

C. Proposed Algorithm-MFCFT

In MFCFT the representation for cubic chirps is given by

$$
\begin{gathered}
X(k, l, m)=\frac{1}{\sqrt{N}} \sum_{n=0}^{N-1} x(n) W_{N}^{k n+(l / N) n^{2}+(m / N) n^{3}} \\
0 \leq k, l, m \leq N-1
\end{gathered}
$$

where $\mathrm{k}, \mathrm{l}$ and $\mathrm{m}$ represent constant frequency, chirp rate and derivative of chirp rate respectively. Here, the parameters $\mathrm{k}, 1, \mathrm{~m}$ are estimated as $\mathrm{k} \epsilon(0,1, \ldots \mathrm{N}-1), 1 \epsilon(0,1 / \mathrm{N}, \ldots(\mathrm{N}-1) / \mathrm{N}), \mathrm{m} \epsilon(0,1 / \mathrm{N}, \ldots(\mathrm{N}-1) / \mathrm{N})$. Therefore it increases the accuracy and near the peak value is identified. This is clearly shown in case (i \& ii).

Case i. If $\mathrm{k}=36, \mathrm{l}=98, \mathrm{~m}=25$ and signal length $\mathrm{N}=127$ then the output for MFCFT is shown in Fig. 4 


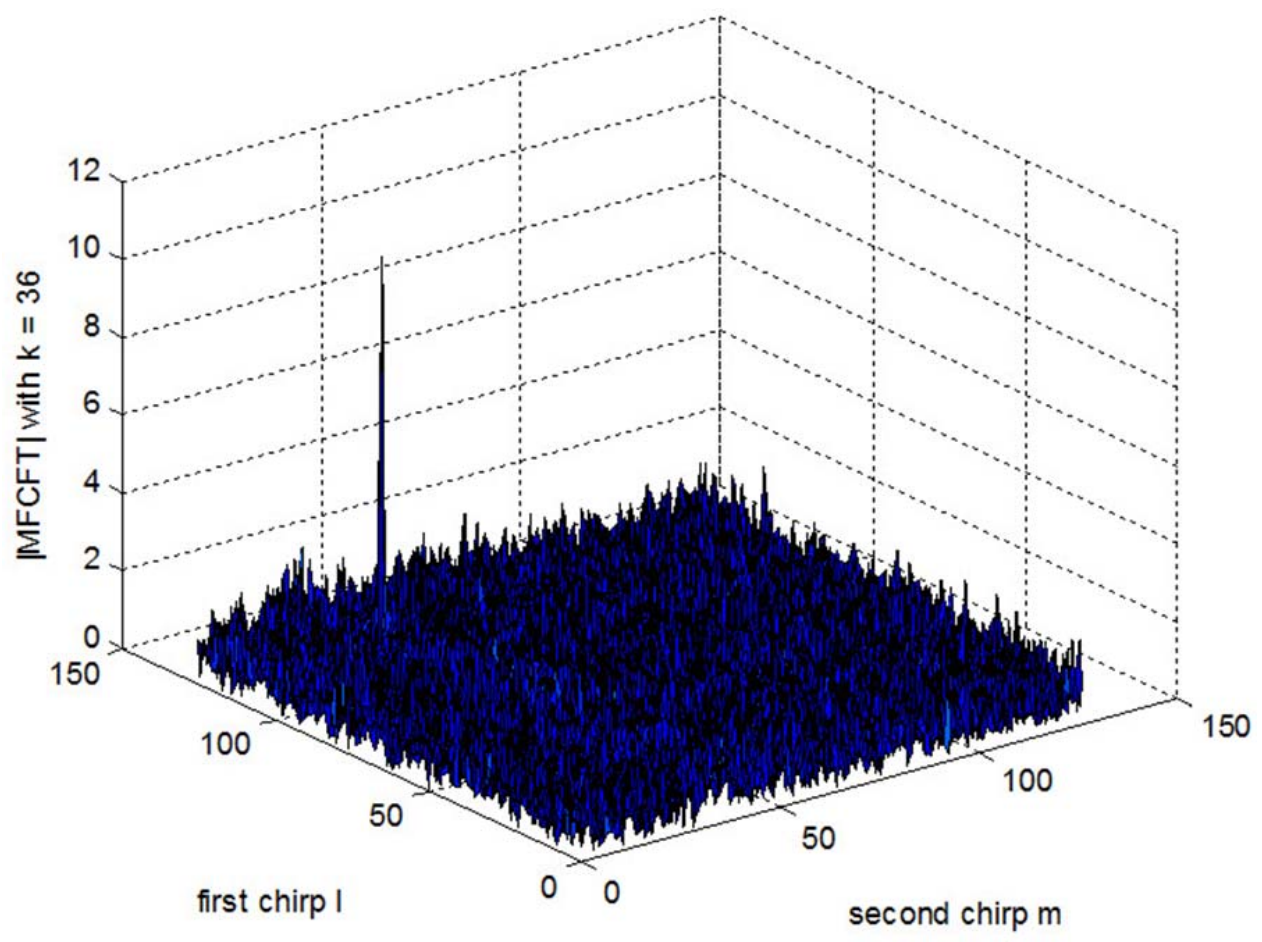

Fig.4.Three dimensional plot for Proposed method MFCFT for $\mathrm{N}=127$

Case (ii). If $\mathrm{k}=36, \mathrm{l}=98$ and $\mathrm{m}=25$ and signal length $\mathrm{N}=128$ then the peak is clearly identified as shown in Fig.5

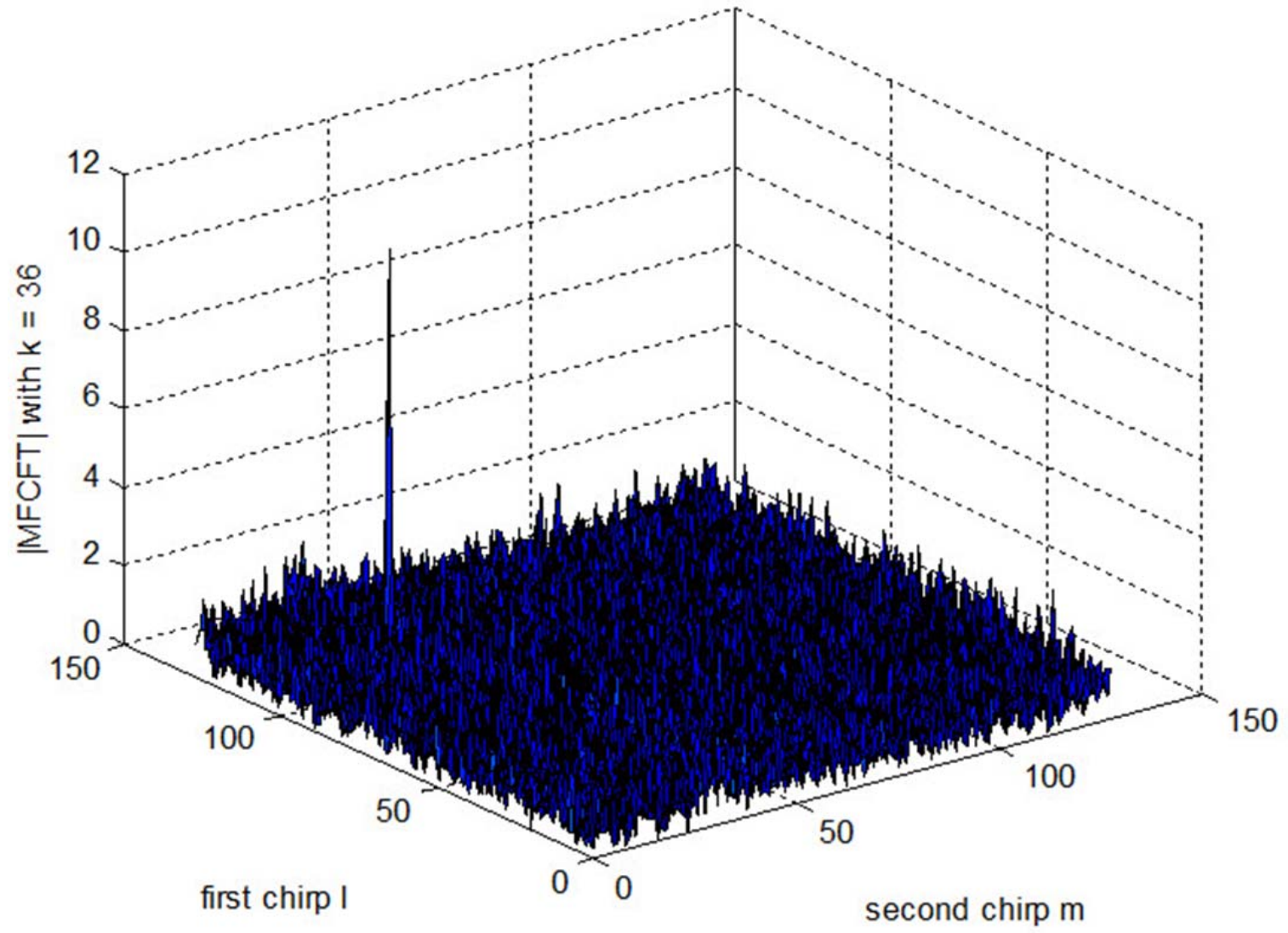

Fig.5. Three dimensional plot for MFCFT for $\mathrm{N}=128$

From the figures one can say that the peak is clearly identified in the two cases irrespective of signal length being prime number or not. The ISAR imaging based on MFCFT for cubic chirps is implemented as follows:

i. MFCFT equation given in (10) is applied to $\mathrm{s}(\mathrm{n})$ in each range bin, to get $\mathrm{S}(\mathrm{k}, \mathrm{l}, \mathrm{m})$ given by 


$$
S(k, l, m)=\frac{1}{\sqrt{N}} \sum_{n=0}^{N-1} s(n) W_{N}^{k n+(l / N) n^{2}+(m / N) n^{3}}
$$

where $\mathrm{S}(\mathrm{k}, \mathrm{l}, \mathrm{m})$ is the MFCFT of $\mathrm{s}(\mathrm{n})$.

ii. The projection of $S(k, 1, m)$ will produce a Doppler image in 3D with center frequency $f_{i}$.

iii. Doppler image $\mathrm{I}_{\mathrm{D}}(\mathrm{k})$ can be obtained by the energy accumulation along axes of 1 and $\mathrm{m}$ given by

$$
I_{D}(k)=\left|\sum_{l, k} S(k, l, m)\right|
$$

In this Doppler image $\mathrm{I}_{\mathrm{D}}(\mathrm{k})$ all the main lobes of $\mathrm{S}(\mathrm{k}, 1, \mathrm{~m})$ are clearly identified.

iv. Now the Doppler images of all range bins are added to form the ISAR image of the target for cubic chirp signal.

v. It may be noted that the proposed algorithm will be found to be more and more effective as higher and higher order terms are considered because exponent of $\mathrm{N}$ is defered with.

In this way the 2D ISAR image is obtained for MFCFT where the computation time is very much reduced when compared to MDCFT.

\section{IV.SIMULATION RESULTS}

The simulation parameters used in the programming are given in Table. I

TABLE.I SIMULATION PARAMETERS

\begin{tabular}{|c|c|c|}
\hline Parameter & Representation & Value \\
\hline Signal length & $\mathrm{N}$ & 150 \\
\hline Bandwidth & $\mathrm{BW}$ & $384 \mathrm{MHz}$ \\
\hline Pulse repetition frequency & prf & $100 \mathrm{~Hz}$ \\
\hline Initial range & $\mathrm{R}_{0}$ & $45 \mathrm{kms}$ \\
\hline Frequency & $\mathrm{f}$ & $5.2 \mathrm{GHz}$ \\
\hline Constant terms & $\omega_{1} \omega_{2} \omega_{3}$ & {$[0.50 .30 .6]$} \\
\hline First order terms & $\alpha_{1} \alpha_{2} \alpha_{3}$ & {$[0.60 .20 .5]$} \\
\hline Second order terms & $\gamma_{1} \gamma_{2} \gamma_{3}$ & {$[0.40 .50 .6]$} \\
\hline No of scatters & --- & 85 and 84 \\
\hline
\end{tabular}

Here the simulation has been carried out initially for a ship target with 85 scattering points and for Airplane target with 84 scattering points.

Case i. Simulated ship target is shown in Fig.6. The amplitudes of all the echoes are considered as equal. ISAR image in 2D obtained using MDCFT is shown in Fig.7 and that using MFCFT is shown in Fig.8. In both the cases, the hot spots are clearly identified, but, in case of MFCFT the simulation time is very much reduced when compared to MDCFT.

Case ii. Here an Airplane with 84 scatters is considered as shown in Fig.9. The ISAR image using MDCFT is shown in Fig.10 and that using MFCFT is shown in Fig.11. Here also the hot spots are observed clearly in the two methods but the computation time is reduced in MFCFT. 


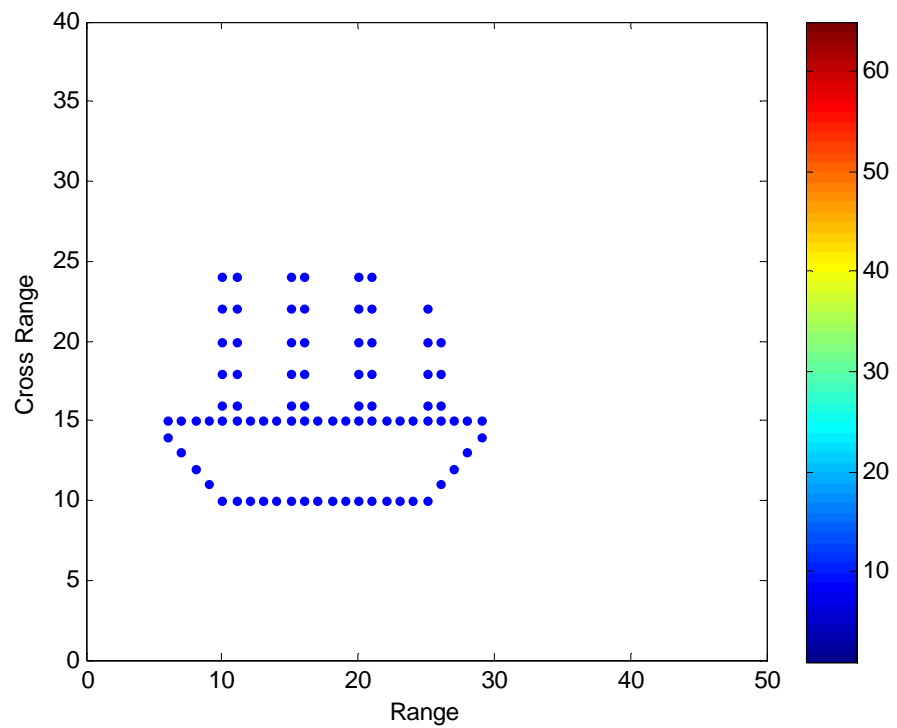

Fig.6 Simulated ship target with 85 scattering points

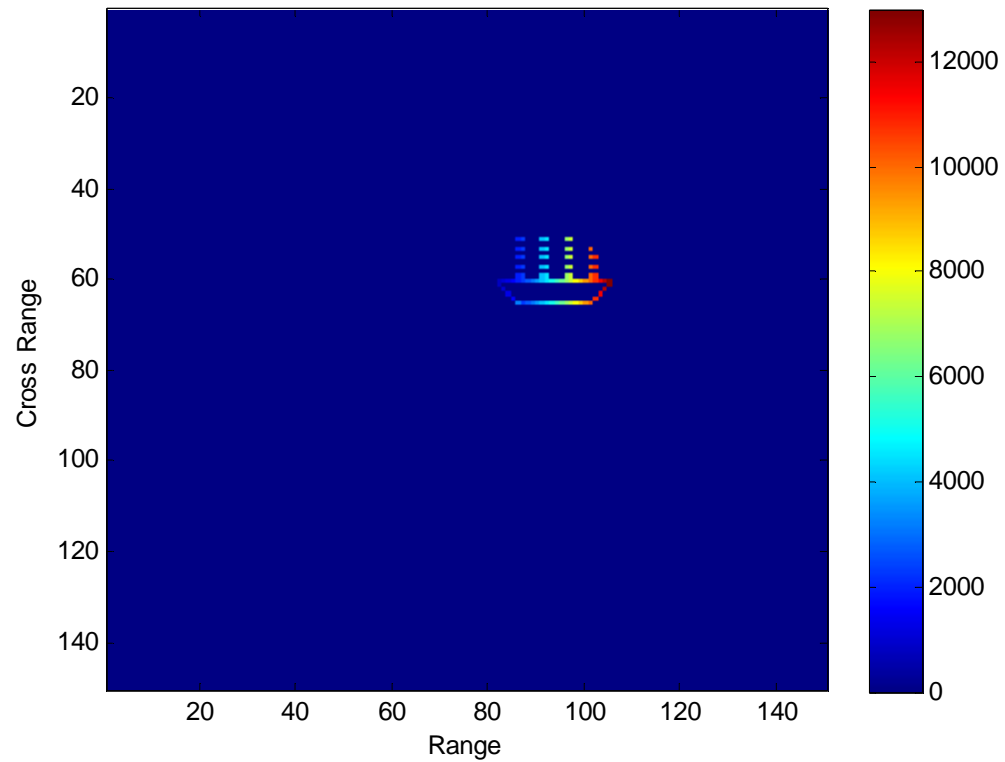

Fig.7. ISAR image of ship target using MDCFT 


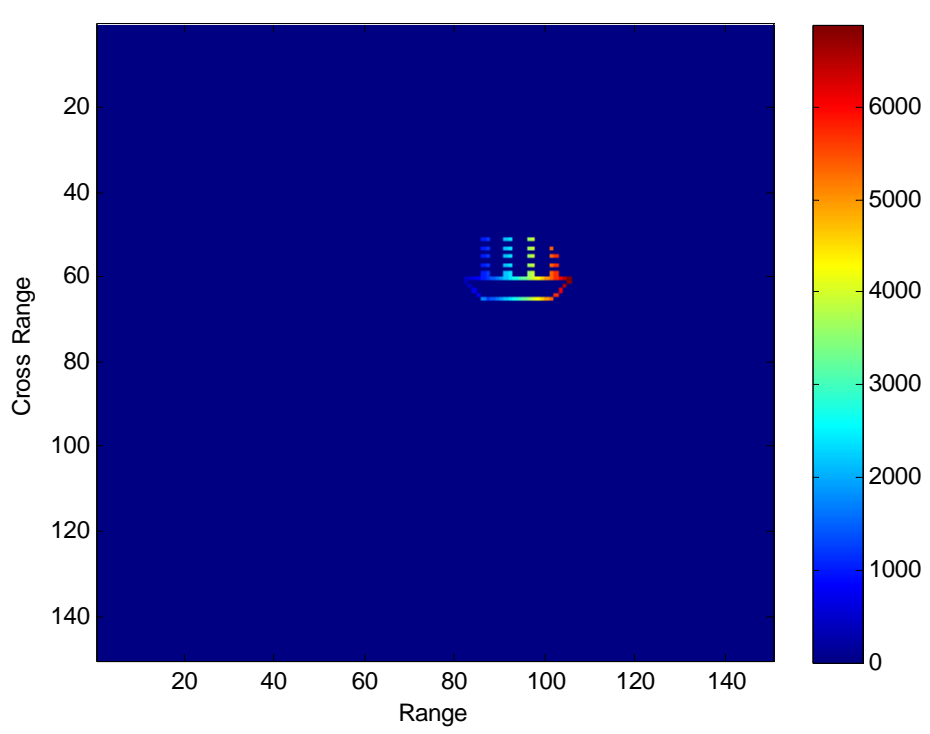

Fig.8. ISAR image of ship target using MFCFT

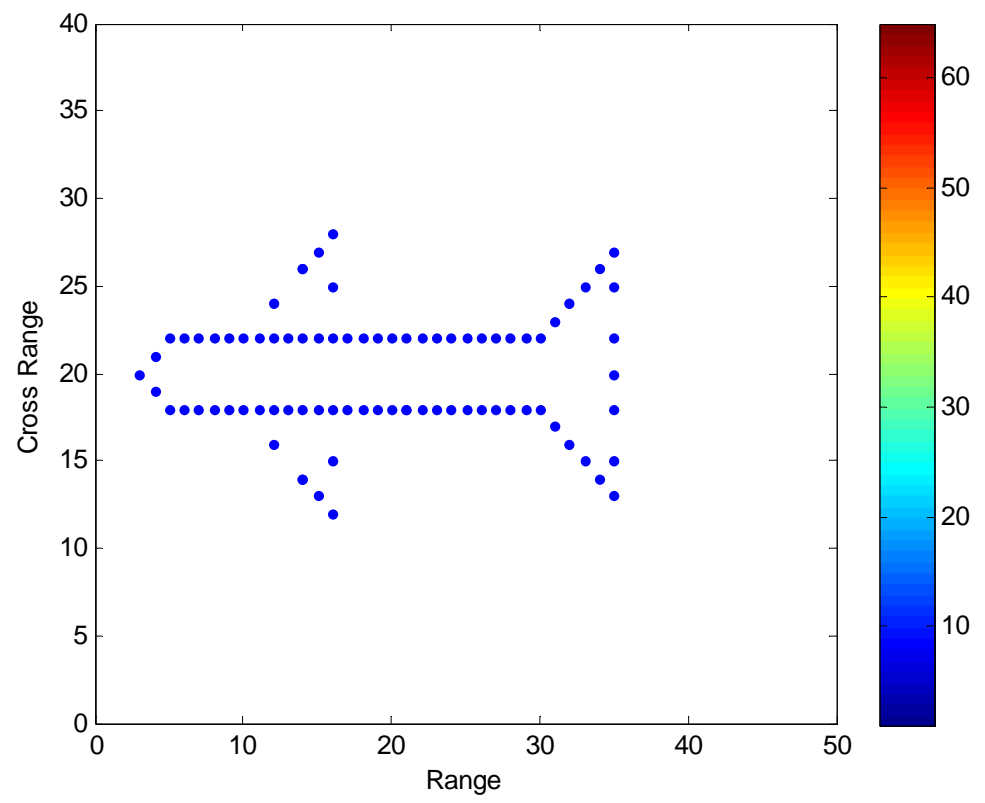

Fig.9 Simulated Airplane target with 84 scattering points 


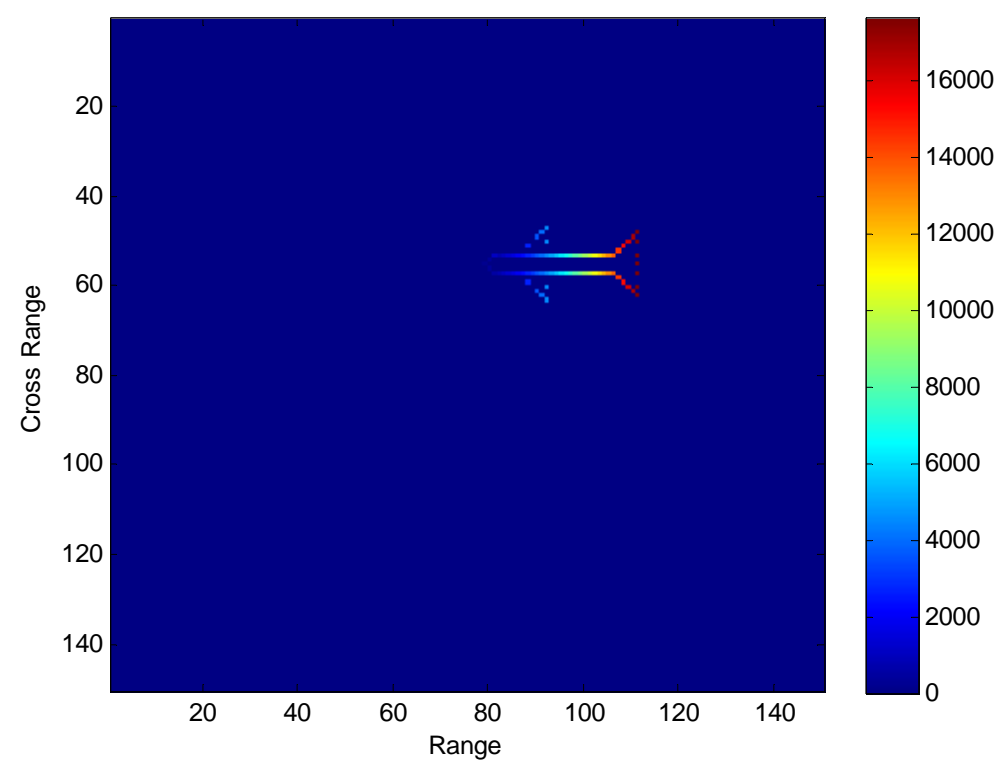

Fig.10. ISAR image of Airplane target using MDCFT

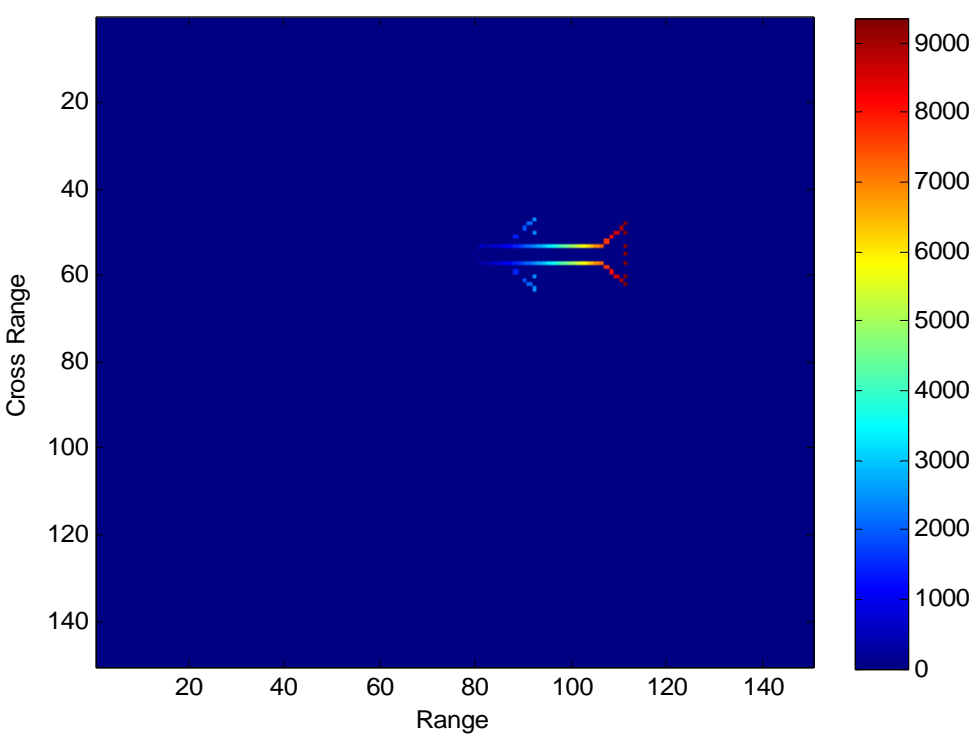

Fig.11.ISAR image of Airplane target using MFCFT

\section{CONCLUSIONS}

In this paper, ISAR imaging using MFCFT is discussed for cubic chirp signals. The quality of the image obtained in MFCFT is comparable with that of MDCFT. Additionally the computation time is significantly reduced here. This method will be found to be more effective as higher and higher order terms need to be considered. The advantage in this method is that exponent terms of the signal length remain to be unity.

\section{REFERENCES}

[1] Wehner D.R, "High Resolution Radar", 2nd ed. Norwood, MA: Artech House, 1995.

[2] F. Berizzi, E. D. Mese, M. Diani, M. Martorella, "High-resolution ISAR imaging of maneuvering targets by means of the range instantaneous Doppler technique: Modeling and performance analysis,” IEEE Trans. Image Pro cess., Vol.10, No.12, pp. 1880-1890, Dec 2001.

[3] V. C. Chen ,S. Qian, “Joint time-frequency analysis for radar range-Doppler imaging,” IEEE Trans. Aerosp. Electron. Syst., Vol 34, No.2, pp. 486-499, April 1998.

[4] G.Wang, Z. Bao,"Inverse synthetic aperture radar imaging of maneuvering targets based on chirplet decomposition," Opt. Eng., Vol.38,No. 9, pp. 1534-1541,Sep1999.

[5] L. Du, G. Su, "Adaptive inverse synthetic aperture radar imaging for non uniformly moving targets," IEEE Geosci. Remote Sens. Lett., Vol.2,No.3, pp. 247-249, July 2005 
[6] Z. Bao, G. Wang, L. Luo, "Inverse synthetic aperture radar imaging of maneuvering targets," Opt. Eng., Vol.37,No.5, pp. 1582-1588, May 1998.

[7] Y.Wang, Y. Jiang, "ISAR imaging of a ship target using product high order matched-phase transform," IEEE Geosci. Remote Sens.Lett., Vol.6, No.4, pp. 658-661, Feb 2009.

[8] X. Guo, H. B. Sun, S. L.Wang," Comments on discrete chirp-Fourier transform and its application to chirp rate estimation," IEEE Trans. Signal Process., Vol. 50, No.12, pp. 3115-3116, Dec 2002.

[9] X.G.Xia, "Response to comments on discrete chirp-Fourier transform and its application to chirp rate estimation," IEEE Trans. Signal Process., Vol. 50 No.12, pp. 3116, Dec 2002.

[10] P. Fan, X.G. Xia, 'Two modified discrete chirp-Fourier transform schemes,' Sci. China, ser. F, Vol.44,No.5, pp. 329-341, June 2001.

[11] Liang Wu, Xizhang Wei, Degui Yang, et.al, "ISAR Imaging of Targets With Complex Motion Based on Discrete Chirp Fourier Transform for Cubic Chirps,” IEEE Trans. Geoscience and Remote Sensing., Vol. 50, No.10, pp.4201-4212, July 2012.

[12] M. D. Xing, R. Wu, Z. Bao, 'High resolution ISAR imaging of high speed moving targets,' Inst. Elect. Eng. Proc. Radar Sonar Navig.,Vol. 152, No. 2, pp. 58-67, April 2005.

[13] Y.Wang, "Inverse synthetic aperture radar imaging of maneuvering target based on range instantaneous Doppler and rangeinstantaneous chirp rate algorithms", IET Radar Sonar Navigation, Vol. 6, Issue. 9, pp.921-928, July 2012.

[14] Wang Ran, Jiang Yi-Cheng, "ISAR ship imaging based on Reassigned Smoothed Pseudo Wigner Ville Distribution", IEEE conference, Oct 2010.

[15] Xiaolei Lv, Mengdao Xing, Chunru Wan, Shou hong Zhang, "ISAR Imaging of Maneuvering Targets Based on the Range Centroid Doppler Technique", IEEE Transactions on Image Processing, Vol.19, No.1, pp.141-153, Jan 2010.

[16] Yong Wang, Yicheng Jiang, "New approach for ISAR imaging of ship target with 3D rotation", Multidim Syst Sign Process Springer Vol. 21, pp.301-318, 2010.

[17] Yong Wang, Yicheng Jiang, "Inverse Synthetic Aperture Radar Imaging of Maneuvering Target Based on the Product Generalized Cubic Phase Function “, IEEE Geoscience and Remote Sensing Letters, Vol. 8, No. 5, pp.958-962, Sep 2011.

[18] Yong Wang, Yi Cheng Jiang, "ISAR Imaging of Ship Target with Complex Motion Based on New Approach of Parameters Estimation for Polynomial Phase Signal”, EURASIP Journal on Advances in Signal Processing, Vol.2011, pp.1-9,Mar 2011

[19] G.Anitha, K.S.Ranga Rao, "Optimization of Parameters for ISAR imaging using MDCFT algorithm and its comparison with Gabor transform", International Journal of Applied Engineering Research, Vol.11, No.3, pp.1556-1561, 2016.

[20] G.Anitha, K.S.Ranga Rao, "Comparison of various Algorithms for RD, RID and RIC methods using ISAR imaging”, IJAER, Vol.11, No.5, pp.3364-3368, 2016.

\section{AUTHOR PROFILE}

Anitha. G graduated in E.C.E from JNTU University in 2006 and did her Master's degree in RF and Microwave from Andhra University in 2008. She is presently working as Assistant Professor in Dept. of E.C.E in GITAM Institute of Technology, GITAM university since 2008 till date. Her areas of interest are Radars, signal processing, etc.

Dr. K. S. RangaRao graduated in Electronics and Communication Engineering from College of Engineering, Kakinada, JNTU Hyderabad in 1977 and Ph.D in Engineering from Electronics and Telecommunication Engineering Department of Jadavpur University, Kolkata in 1994. After having been in IIT, Kharagpur from 1982 to 1996, he joined as a Scientist in Integrated Test Range, Chandipur from 1997 to 2006. He then worked with Naval Science and Technological Laboratory, Visakhapatnam from 2006 to 2015. He is presently working as Professor in the Department of ECE in ANITS Engineering College. His area of work includes RF and Microwave Engineering, Antennas, RADAR \& LASER Engineering. 